\title{
Bioengineering and Enabling Technologies: ABME Special Issue Editorial
}

(Published online 30 March 2020)

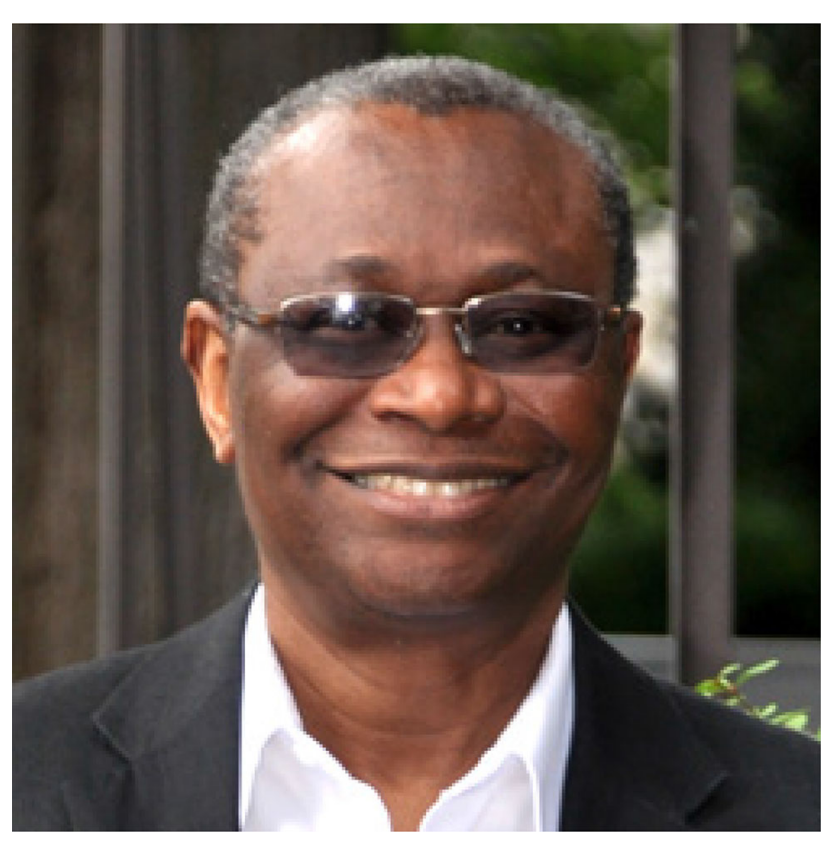

Emmanuel C. Opara

Tissue engineering, a major component of the translational research discipline called regenerative medicine, deals with the process of engineering and replacing damaged tissues and organs in order to restore function. In tissue engineering, biomaterials, cells and bioactive factors are the major ingredients. In a recent book, Definitions of Biomaterials for the TwentyFirst Century by Xingdong Zhang and David F. Williams, biomaterial is defined as "a material designed to take a form that can direct, through interactions with living systems, the course of any therapeutic or diagnostic procedure." In addition, Zhang and Williams define tissue engineering as "the use of cells, biomaterials and suitable molecular or physical factors, alone or in combination, to repair or replace tissue to improve clinical outcomes". ${ }^{10}$

In the context of this special issue of ABME, tissue and organ bioengineering may be described as: the intersection of biomaterials, cells, and techniques, as illustrated in Fig. 1. The cells could be either primary or stem cells. Thus, in this journal issue, we are highlighting the roles played by biomaterials, techniques, biological cells and bioactive factors in the fabrication of tissues for replacement, repair, or regeneration of damaged tissues. Of significant note in this issue is the fabrication of unique biomaterial scaffolds for various tissue engineering applications. It is also noteworthy that the term "enabling technologies" is used in lieu of the word techniques in the title of this journal special issue because of the wider subject breadth that the former encompasses compared to the latter that is more routinely used to describe a specific procedure. However, to keep it simple both terms are used interchangeably in this discussion. Among the enabling technologies presented, the paper by Enck, $\mathrm{K}$ et al. from the Wake Forest institute for Regenerative Medicine (WFIRM) describes a new microfluidic approach for alginate hydrogel-based cell encapsulation, which is a prominent technique in tissue engineering. In the paper, Kevin Enck and his colleagues use a rapid prototyping approach incorporating patterned adhesive thin films to develop a reusable microfluidic device that can produce alginate hydrogel microbeads with high-throughput potential for microencapsulation applications.

To illustrate the role of cell encapsulation in tissue engineering, there is an elegant paper from Jennifer West's lab at Duke that describes the culture of adipose tissue-derived stem cells (ADSCs) in basal media or pro-angiogenic media supplemented with VEGF in 2D prior to their encapsulation at either low or high densities within PEG-based hydrogel for vascular tissue engineering. The encapsulated cells were maintained in either basal media or pro-angiogenic media. The cells were then isolated from the hydrogels and cultured in Matrigel to assess the potential for tubule formation. This work shows that maintenance of ADSCs in a pro-angiogenic medium in $2 \mathrm{D}$ monoculture alone does not result in any CD31 expression. Furthermore, the level of CD31 expression was affected by the density of the cells encapsulated within the PEG-based hydrogel. Upon isolation of these cells, the investigators found that the induced ADSCs were able to form tubules within Matrigel, indicative of endothelial function, while ADSCs cultured in basal 

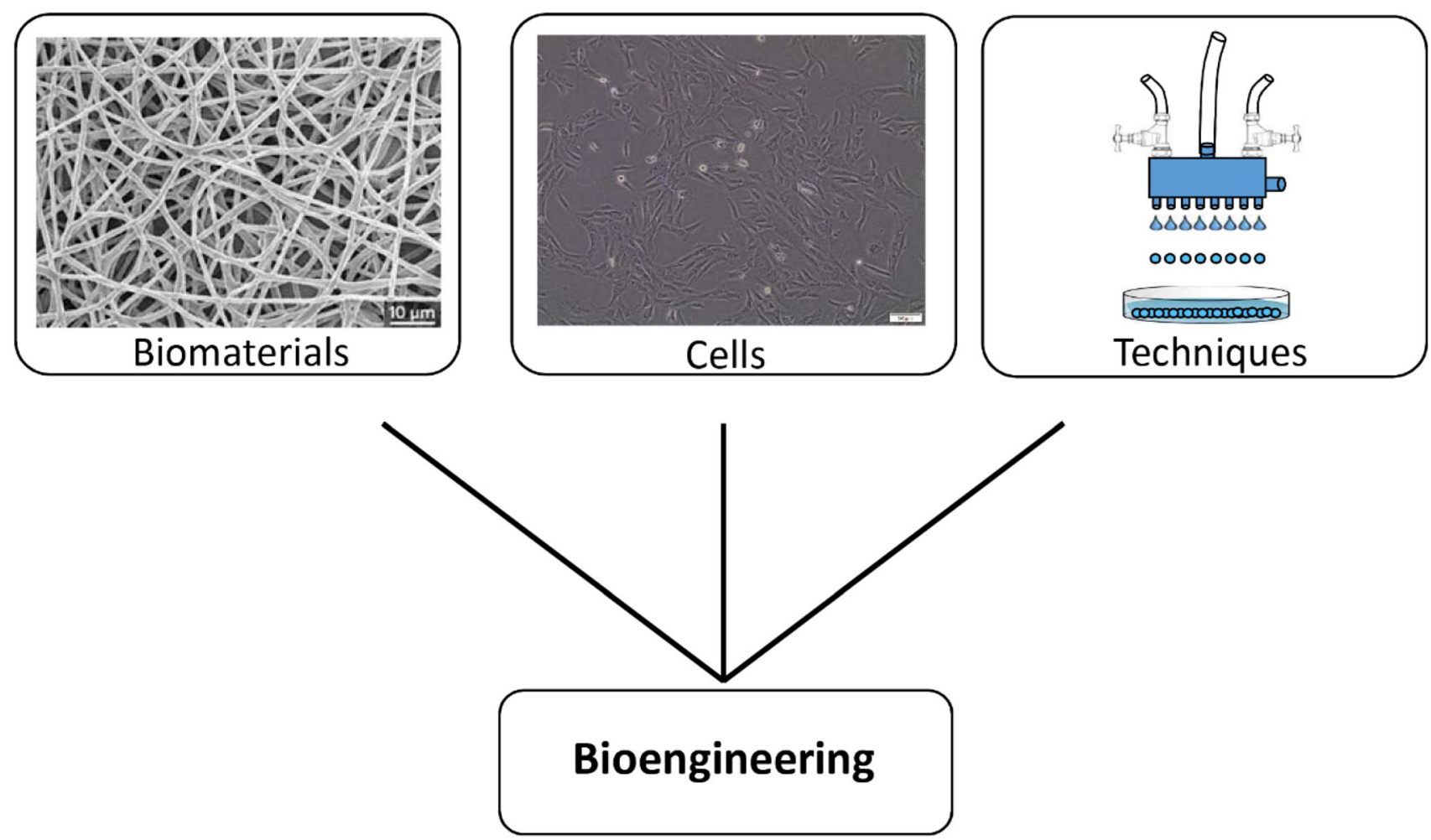

FIGURE 1. Illustration of bioengineering as the intersection of biomaterials, cells, and techniques.

medium could not. This finding points to the potential for the ADSCs to serve as a safe and reliable source of endothelial cells for tissue engineering and regenerative medicine purposes.

In a related paper, the Mequanint lab at the University of Western Ontario, London, Canada in collaboration with their colleagues at the University of Manitoba, Winnipeg, Canada describe the fabrication of tubular electrospun poly(ester amide) (PEA) fibers from L-phenylalanine-derived biodegradable biomaterials seeded with mouse embryo multipotent mesenchymal progenitor cells $(\mathrm{C} 3 \mathrm{H} / 10 \mathrm{~T} 1 / 2$ cells). They investigated cell-scaffold interactions as well as their differentiation into vascular smooth muscle cell and subsequent elastin expression. PEA scaffolds fabricated under different collector speeds did not have an impact on the fiber directionality/orientation. 10T1/2 cytocompatibility and proliferation studies showed that PEA fibres were not cytotoxic and were able to support proliferation for 14 days. Furthermore, cells were observed infiltrating the fibrous scaffolds despite the small pore sizes $(\sim 5 \mu \mathrm{m})$. Vascular differentiation studies of $10 \mathrm{~T} 1 / 2$ cells using qPCR, Western blot, and immunostaining showed a TGF $\beta 1$-induced upregulation of vascular smooth muscle cell (VSMC)-specific markers smooth muscle alpha-actin (SM- $\alpha$-actin) and smooth muscle myosin heavy chain (SM-MHC). Differentiated $10 \mathrm{~T} 1 / 2$ cells produced both elastin and fibrillin-1 suggesting the potential of fibrous PEA scaffolds to fabricate model vascular tissues.

Currently, the most used stem cell source for tissue engineering applications in both experimental studies and clinical trials is the bone marrow-derived mesenchymal stem cells (BMSCs). ${ }^{2}$ Hence, the paper by Sittadjody et al. at WFIRM in collaboration with Justin Saul at Miami University, $\mathrm{OH}$, examined if hormone secretion from their encapsulated ovarian tissue cell-based hormone replacement (cHT) constructs are impacted by incorporation of BMSCs since these cells contain regulatory factors such as aromatase necessary for estrogen production. Incorporation of BMSCs into the constructs led to enhanced estrogen secretion in vitro. Moreover, cHT constructs with BMSCs achieved estrogen secretion levels significantly greater than constructs without BMSCs in ovariectomized rats from 70 to 90 days after implantation, while also regulating the pituitary hormones, $\mathrm{LH}$ and FSH. cHT constructs with BMSC ameliorated estrogen deficiency-induced uterine atrophy without causing hyperplasia. The results indicate that inclusion of BMSCs in cHT strategies can improve construct performance. This work represents yet another illustration 
of the use of the technique of cell microencapsulation in tissue engineering. ${ }^{7}$

In addition to the stem cell types used in the abovementioned studies, a review article by Padma Rajagopalan at Virginia Tech provides an overview of the biology and utilization of another stem cell source, the induced pluripotent stem cells (iPSCs). There is significant interest in iPSCs as they are perceived to be an alternative source of pluripotent stem cells without the ethical concerns associated with the use of the embryonic stem cells, albeit, they both pose the risk of teratomas. ${ }^{8}$ iPSCs can be differentiated into multiple cell types in the body while maintaining proliferative capabilities. The generation of hepatocyte-like cells (HLCs) from iPSCs has resulted in a new source of liver cells. This review focuses on advances being made to achieve hepatic functions from HLCs, their current use in hepatotoxicity testing, and their potential for future liver-related toxicity evaluations.

A paper by Aleks Skardal and his colleagues describes an engineered in vitro colorectal cancer $3 \mathrm{D}$ micro-tumor construct for cell lines and patient biospecimens suitable for standard-of-care and experimental drug screening. In their work, they utilized an extracellular matrix (ECM)-derived hydrogel to create a 3D micro-tumor construct platform capable of both supporting cells for long time durations and for high throughput drug screening. In a related review article that highlights the emerging role of ECM in tissue engineering, Amrinder Nain and his associate at Virginia Tech provide an elegant summary of details about the native architecture, composition, and mechanical properties of the in vivo ECM at the early embryonic stages and the later adult stages. Native ECM from adult tissues categorized on their origin from respective germ layers are discussed while engineering techniques employed to facilitate differentiation of stem cells into particular lineages are noted. Overall, the authors emphasize that in vitro strategies need to integrate tissue specific ECM biophysical cues for developing accurate artificial environments in order to optimize stem cell differentiation.

A promising enabling technology suitable for application in monitoring the in vivo performance of implanted tissue engineered constructs is the $\mathrm{x}$-ray Phase Contrast (XPC) presented in this issue by Eric Brey and his team from UT San Antonio and other collaborators. In their study this group used XPC tomography to image alginate hydrogel microspheres within a rat omentum pouch model with a commercially available $\mathrm{x}$-ray source. Multilayer microbeads could be identified in the XPC images with volumetric and structural information not possible in histological analysis. This study show that XPC tomography can be a useful tool for monitoring of implanted soft biomaterials in small animal models.

A paper at the interface of enabling technologies and biomaterials is presented by an interdisciplinary research team from the University of Central Florida in Orlando. This group describe a mathematical model integrating Computational Fluid Dynamics with Population Balance Model to predict average pore size (APS) of 3D porous chitosan-alginate scaffolds and to assess the influence of the geometrical parameters of mold on scaffold pore structure. The model predicted the crystallization pattern and APS for scaffolds cast in different diameter molds and filled to different heights. The predictions demonstrated that the temperature gradient and solidification pattern affect ice crystal nucleation and growth, subsequently influencing APS homogeneity. The predicted APS compared favorably with APS measurements from a corresponding experimental dataset, thus validating the model. The authors suggest that this validated model represents a method for optimizing the APS of freeze-cast biomaterial scaffolds that could be applied to other compositions or applications.

In the biomaterials domain, another interdisciplinary team lead by Elizabeth Cosgriff-Hernandez presents an interesting paper dealing with resorbable hydrogels that have numerous potential applications in tissue engineering and drug delivery due to their highly tunable properties and soft tissue-like mechanical properties. The incorporation of esters into the backbone of poly(ethylene glycol) hydrogels has been used to develop libraries of hydrogels with tunable degradation rates. However, these synthetic strategies used to increase degradation rate often result in undesired changes in the hydrogel physical properties such as matrix modulus or swelling. In an effort to decouple degradation rate from other hydrogel properties, the investigators inserted thio- $\beta$ esters into the poly(ethylene glycol)-diacrylate backbone to introduce labile bonds without changing macromer molecular weight. This allowed the number of hydrolytically labile thio- $\beta$ esters to be controlled through changing the ratios of this modified macromer to the original macromer without affecting network properties. The retention of hydrogel properties at different macromer ratios was confirmed by measuring gel fraction, swelling ratio, and compressive modulus. The tunable degradation profiles were characterized both in vitro and in vivo. Following confirmation of cytocompatibility after exposure to the hydrogel degradation products, the in vivo host response was evaluated in comparison to medical grade silicone. Collectively, this work demonstrates the utility and tunability of these hydrolytically degradable hydrogels for a wide variety of tissue engineering applications.

BMES Sowewew 
This special issue of ABME particularly highlights various advances in bone tissue engineering that include the use of unique biomaterials and cells because of the increasing global incidence of bone disorders and conditions associated with increases in aging populations. ${ }^{1,4}$ Susmita Bose and her associate present one paper whose objective was to understand the effects of random and controlled scaffold porosity on the release kinetics of vitamin $\mathrm{D}_{3}$ in order to determine if a designed porous structure was comparable in effectiveness on osteoblast proliferation to the randomized essence of natural bone. In this study, porous tricalcium phosphate (TCP) scaffolds were prepared by fugitive material removal method using naphthalene and $3 \mathrm{D}$ printing to model random and controlled porosity, respectively. Scaffold comparison was made based on open pore volume percentage of which naphthalene scaffolds had $45.8 \pm 1.5 \%$ and $3 \mathrm{D}$ printed scaffolds had $48.9 \pm 2.5 \%$. Comparative analysis of traditional bioceramic processing to additive manufacturing was limited especially regarding drug release kinetics. The results showed that the naphthalene scaffold surface area was only $0.3 \%$ that of $3 \mathrm{D}$ printed scaffolds due to the lower open pore interconnectivity. This increase in surface area produced higher release of drug and osteoblast proliferation in $3 \mathrm{D}$ printed scaffolds comparatively. By 11 days, osteoblast proliferation was enhanced by $64 \%$ in scaffolds manufactured using $3 \mathrm{D}$ printing compared to traditional processing. The investigators conclude that understanding the effects of processing methods of TCP scaffolds on the release kinetics of vitamin $\mathrm{D}_{3}$ and the system effects on cells can aid in low load bearing applications for bone tissue engineering.

In another paper, a team led by Syam Nukavarapu at the University of Connecticut evaluated an engineered hybrid matrix for bone regeneration via endochondral ossification. Despite its regenerative ability, long and segmental bone defect repair remains a significant orthopedic challenge. Conventional tissue engineering efforts induce bone formation through intramembranous ossification (IO) which limits vascular formation and leads to poor bone regeneration. To overcome this challenge, a novel hybrid matrix comprised of a load-bearing polymer template and a gel phase was designed and assessed for bone regeneration. In previous studies the investigators had developed a synthetic ECM, hyaluronan (HA)-fibrin (FB), that was able to mimic cartilage-mediated bone formation in vitro. In the present study, the wellcharacterized HA-FB hydrogel was combined with a biodegradable polymer template to form a hybrid matrix. In vitro evaluation of the matrix showed car- tilage template formation, cell recruitment and recruited cell osteogenesis, which are essential stages in endochondral ossification. A transgenic reportermouse critical-defect model was used to evaluate the bone healing potential of the hybrid matrix in vivo. The results demonstrated host cell recruitment into the hybrid matrix that led to new bone formation and subsequent remodelling of the mineralization. Overall, this study developed and evaluated a novel loadbearing graft system for bone regeneration via endochondral ossification.

In the paper presented by Aaron Goldstein and his associate, they described a method to improve the osteogenicity of PCL fiber substrates by surface-immobilization of bone morphogenic protein-2 (BMP-2). PCL fiber scaffolds are attractive, albeit inert, substrates for ligament regeneration that may be improved by incorporating trophic factors to guide tissue remodelling in vivo. In particular, immobilization of bone morphogenic protein-2 (BMP-2) to the scaffold surface may facilitate rapid and robust integration of the scaffold with adjacent bone tissues. As a first step toward testing this, model PCL surfaces were modified by the addition of heparin (Hep) and BMP-2 to facilitate osteoblastic differentiation. Specifically, Hep was combined with PCL at $0,0.5$, and $1 \mathrm{wt} \%$ (denoted as PCL, PCL-0.5Hep, and PCL-1Hep), cast into films, and then BMP-2 was immobilized to surfaces by either adsorption and covalent conjugation. Here, BMP-2 concentration increased systematically with incorporation of Hep, and higher concentrations were achieved by covalent conjugation. Next, blends were electrospun to form thin meshes with fiber diameters of $0.92,0.62$, and $0.54 \mu \mathrm{m}$ for PCL, PCL- $0.5 \mathrm{Hep}$, and PCL-1Hep, respectively. Mesenchymal stem cells (MSCs) had no difficulty attaching to and proliferating on all meshes. Lastly, PCL-1Hep meshes were prepared with adsorbed or covalently conjugated BMP-2 and cultured with MSCs in the absence of osteogenic factors. Under these conditions, alkaline phosphatase activity and deposition of bone sialoprotein, osteopontin, and calcium minerals - markers of osteoblastic differentiation - were significantly higher on surfaces with immobilized BMP-2. Together, these data indicate that covalent immobilization of trophic factors confers bioactivity to scaffolds, which may be applied in a spatially controlled manner for ligament regeneration and bone integration.

A multi-institutional research team led by Regis O'Keefe at Washington University in St. Louis, MO presents a paper showing that the inhibition of the prostaglandin EP-1 receptor in periosteum progenitor cells enhances osteoblast differentiation and fracture 
repair. Fracture healing is a complex and integrated process that involves mesenchymal progenitor cell (MPC) recruitment, proliferation and differentiation that eventually results in bone regeneration. Prostaglandin E2 (PGE2) is an important regulator of bone metabolism and has an anabolic effect on fracture healing. Prior work from the O'Keefe laboratory showed that $\mathrm{EP}^{-1-}$ mice have enhanced fracture healing, stronger cortical bones, higher trabecular bone volume and increased in vivo bone formation. The investigators also showed that bone marrow MSCs from $\mathrm{EP}^{-/-}$mice exhibit increased osteoblastic differentiation in vitro. In the present study, they investigated the changes in the periosteal derived MPCs (PDMPCs), which are crucial for fracture repair, upon EP1 deletion. EP1 ${ }^{-/-}$PDMPCs exhibit increased numbers of total (CFU-F) and osteoblastic colonies (CFU-O) as well as enhanced osteoblastic and chondrogenic differentiation. Moreover, they tested the possible therapeutic application of a specific EP1 receptor antagonist to accelerate fracture repair. Their findings showed that EP1 antagonist administration to wild type mice in the early stages of repair similarly resulted in enhanced CFU-F, CFU-O, and osteoblast differentiation in PDMPCs leading to enhanced fracture callus formation at 10 days post fracture, and increased bone volume and improved biomechanical healing of femur fractures at 21 days post fracture.

In this journal issue, we also include promising technologies to address other musculoskeletal disorders that require engineering of tendons and meniscus, which are important for a wide spectrum of disabilities. ${ }^{3,5,6}$ Thus, in one paper that overlaps between enabling technologies and tissue engineering, the Lovati laboratory in Milan, Italy describe an independent, controllable stretch-perfusion bioreactor chambers to functionalize cell-seeded decellularized tendons. The purpose of their study was to develop and validate a custom-made automated bioreactor, called oscillating stretch-perfusion bioreactor (OSPB), consisting of multiple, independent culture chambers able to combine a bidirectional perfusion with a programmable, uniaxial strain to functionalize cell-seeded decellularized tendons. Decellularized tendon matrices were seeded on their surfaces and within the tendon fibers with mesenchymal stem cells. Then, they were subjected to a bidirectional perfusion and programmed stretching cycles of 15-30-60 min on-off two times per day for 7 days of culture. In vitro analyses showed viable cells, homogenously distributed on the surface of the constructs. More importantly, cell-seeded decellularized tendon grafts undergoing cyclic load in the bioreactor had a superior production and organization of newly formed collagen matrix compared to static cultured constructs. This system allows parallel pro- cessing of several customized tendon constructs to be used as grafts to enhance the surgical repair of large tendon defects. This paper highlights the important role of perfusion bioreactors for cell cultures and engineered tissue because of their ability to increase nutrient supply to cells throughout scaffolds compared to static conditions. ${ }^{9}$

In another paper, Adetola Adesida and his colleagues at the University of Calgary, Alberta, Canada and others in Guangdong, People's Republic of China report that the re-differentiation of human meniscus fibrochondrocytes differs in three-dimensional cell aggregates and decellularized human meniscus matrix scaffolds. The investigators used human meniscus-derived decellularized matrix (DCM) to re-differentiate human meniscus fibrochondrocytes (MFCs) to form meniscus-like extracellular matrix (ECM). They expanded human MFCs and seeded them upon a cadaveric meniscus-derived DCM prepared by physical homogenization under hypoxia. To assess the bioactivity of the DCM, they used conditions with and without chondrogenic factor TGF- $\beta 3$ and set up a cell pellet culture model as a biomaterial-free control. They found DCM supported chondrogenic re-differentiation and ECM formation of MFCs only in the presence of exogenous TGF- $\beta 3$. Chondrogenic redifferentiation was more robust at the protein level in the pellet model as MFCs on the DCM appeared to favour a more proliferative phenotype. Interestingly, without growth factors, the DCM tended to promote expression of hypertrophic differentiation markers relative to the pellet model. The investigators concluded that the human meniscus-derived DCM prepared by physical homogenization contained insufficient bioactive factors to induce appreciable ECM formation by human MFCs.

\section{REFERENCES}

\footnotetext{
${ }^{1}$ Amini, A. R., C. T. Laurencin, and S. P. Nukavarapu. Bone tissue engineering: recent advances and challenges. Crit Rev Biomed Eng 40(5):363-408, 2012.

${ }^{2}$ Domaszewska-Szostek, A., M. Krzyżanowska, and M. Siemionow. Cell-based therapies for chronic wounds tested in clinical studies: review. Ann. Plast. Surg. 83(6):e96-e109, 2019. https://doi.org/10.1097/SAP.0000000000001947.

${ }^{3}$ Dzobo, K., K. S. C. M. Motaung, and A. Adesida. Recent trends in decellularized extracellular matrix bioinks for 3D printing: an updated review. Int. J. Mol. Sci. 20(18):E4628, 2019. https://doi.org/10.3390/ijms20184628.

${ }^{4}$ Hadjiar, M., and R. J. O'Keefe. The convergence of fracture repair, and stem cells: interplay of genes, aging, environmental factors and disease. J. Bone Miner. Res. 29(11):2307-2322, 2014.
} 
${ }^{5}$ Makris, E. A., D. J. Responte, N. K. Paschos, J. C. Hu, and K. A. Athanasiou. Developing functional musculoskeletal tissues through hypoxia and lysyl oxidase-induced collagen cross-linking. Proc. Natl. Acad. Sci. USA 111(45):E4832-E4841, 2014; (Erratum in: Proc Natl Acad Sci USA 2015 Jun 2; 112(22):E2978).

${ }^{6}$ Martin, J. T., A. H. Millby, K. Ikuta, S. Poudel, C. G. Pfeifer, D. M. Elliott, H. E. Smith, and R. L. Mauck. A radiopaque electrospun scaffold for engineering fibrous musculoskeletal tissues: scaffold characterization and in vivo applications. Acta Biomater. 26:97-104, 2015.

${ }^{7}$ Opara, E. C. Applications of cell microencapsulation. Methods Mol. Biol. 1479:23-39, 2017.

${ }^{8}$ Path, G., N. Perakakis, C. S. Mantoros, and J. Seufert. Stem cells in the treatment of diabetes mellitus - focus on mesenchymal stem cells. Metabolism 90:1-15, 2019.

${ }^{9}$ Santoro, M., S. E. Lamhamedi-Cherradi, B. A. Menegaz, J. A. Ludwig, and A. G. Mikos. Flow perfusion effects on three-dimensional culture and drug sensitivity of Ewing sarcoma. Proc. Natl. Acad. Sci. USA 112(33):100304100309, 2015.
${ }^{10}$ Zhang, X., and D. Williams (eds.). Definitions of Biomaterials for the Twenty-First Century. MaterialsToday: Elsevier, 2020.

\section{Emmanuel C. Opara}

Wake Forest Institute for Regenerative Medicine, Virginia Tech-Wake Forest School of Biomedical Sciences \& Engineering (SBES)

Wake Forest School of Medicine

Electronic mail: eopara@wakehealth.edu

Publisher's Note Springer Nature remains neutral with regard to jurisdictional claims in published maps and institutional affiliations. 